\title{
Emotional investment in a distracting external focus as self-support in addiction therapy for schizophrenic and schizoaffective patients and others
}

\author{
Gillian R M Steggles* \\ University College London Alumna, UK
}

\begin{abstract}
When addiction coincides with independent psychopathology the therapeutic approach can be challenging. However, it is believed that psychosis is more often a result of addiction than vice versa. Therefore, it may be appropriate to treat an addiction initially, and then tackle the psychotic illness. Commonly, a combination of CBT and motivational interviewing is used, but is not described herein.

It has been found that administering a mild electric current to the back of the head can be helpful in alleviating chronic withdrawal symptoms associated with addiction treatment; or drugs may be used for this purpose.

The Psychodynamic Pentapointed Cognitive Construct (PPCC) Theory of understanding how psychotic illnesses may be treated psychodynamically theoretically suggests that developing a strong attachment for an emotionally invested Representation of admired and selected values may be a way forward for an addict, whether or not he or she has an underlying psychopathology. This position is supported by the Alcoholics Anonymous (AA) organization, where God is represented by high ideals of goodness. It is proposed herein that other addicts might benefit from committing themselves to other specific aspects of the world, like goodness, that may be equally well represented by helpful attributes eg. colour and artwork, music and its cultures, or animal contacts and welfare, in their personal "Cherie" or Distracting Investment.

The place of ample support from social and community sources, with generous nurturing and inspiration to invest in their chosen Cherie, is emphasized. It is hoped that (i) (a) drug-free addiction resolution without "cold turkey" effects or alternatively drug therapy, (b) the introduction of an intensely positive alternative focus other than an addictive drug, and then (ii) Psychiatric treatment of the underlying psychosis, may theoretically be a treatment regime that could help these severely ill patients. Perhaps their Cherie could also help them later in their treatment.
\end{abstract}

\section{Introduction}

Addicts may experience terrible difficulties in managing their lives due to their destructive habit. Those addicts who have, in addition, a diagnosable mental illness such as schizophrenia or schizoaffective disorder have even more to contend with. A high proportion of schizophrenic patients smoke (61\%) compared with the normal population (33\%), in findings from the clinical records of 1.7 million people by the Schizophrenia Commission Report in 2012 [1]. The same source found that $26.1 \%$ of schizophrenic patients misused alcohol compared with $11.9 \%$ of healthy people, and $17.8 \%$ of schizophrenic patients abused other substances compared with only $7.0 \%$ of the general population. The combination of severe mental illness and addiction gives these patients a severe challenge if they are ever to regain their mental health.

Psychosis is generally thought to be secondary to addiction when both co-exist [2]. Robin Murray and colleagues have written in a recent paper [3] of the increasing risk for psychosis of traditional marijuana, high-potency cannabis and synthetic cannabinoids, illustrating this direction of the association between addiction and psychosis. In general, treatment of the addiction first is therefore recommended, for example by applying a very mild electric current to the back of the head [4], or alternatively, this may be helped with drugs; then treatment of the mental illness may have a better chance of success, without either drug "highs" or ill effects or withdrawal symptoms. The psychological therapy best suited to the patient's individual needs is then selected, and the patient encouraged to persevere with that in the absence of addiction problems.

The Psychodynamic Pentapointed Cognitive Construct (PPCC) Theory [5] is derived from original data obtained in 1982, and describes the development of the schizoaffective or schizophrenic mind in psychoanalytic psychotherapy from a psychotic state to resolved normality. Its variables, apart from the Problems variable, were derived directly from original data, and coincide exactly with the four variables identified by the Shorter Oxford Textbook of Psychiatry in 2006 [6] as providing the data upon which psychoanalytic theories mainly depend. The PPCC model offers an understanding of the schizophrenic mind, which is characterized by psychosis, and supports the findings of $\mathrm{Dr}$ Michael Robbins [7], who treated a series of 18 schizophrenic patients

Correspondence to: Gillian R M Steggles, University College London Alumna, UK, Email: gill.steggles@btopenworld.com

Key words: addiction, emotional investment, mild electric current, PPCC Theory, schizoaffective disorder, schizophrenia

Received: November 29, 2016; Accepted: December 23, 2016; Published: December 27, 2016 

others

with psychoanalytic psychotherapy, half of whom had a positive outcome to therapy. The PPCC Theory may be adapted to understand the psychology of addiction before the schizophrenic or schizoaffective patient receives treatment. When this psychology is understood, treatment may best be arranged accordingly.

\section{Method}

It has been found [4] that, using a very low electric current applied to acupressure points on the back of the head, the production of natural opioids can be stimulated and the body encouraged to release its own opioids, endorphins (which are suppressed by drugs of addiction eg. nicotine and alcohol) once more. This can lead to a "feel good factor" again, naturally, and can be used to alleviate chronic withdrawal symptoms. Alternatively, specific drugs may be used to alleviate withdrawal symptoms.

The PPCC model illustrates, using diagrams, the structure of the psychotic mind in different states, from the schizophrenic, depressed and manic episodes of schizoaffective disorder to the paranoid-schizoid position [8] and depressive position [9], as identified by Melanie Klein, and to the final, rounded phase reached at the therapeutic termination of therapy. The paranoid-schizoid position is a state of mind where one's appreciation of another person is split into two perspectives; either the person appears wholly good, or they appear wholly bad and nasty. The depressive position is a state of mind where these two perspectives are present at the same time, and one tries to make amends to the other person for hurt we may have caused to them, eg. in thinking badly of them. The PPCC model confirms the findings of Dr Michael Robbins that psychoanalytic psychotherapy may resolve schizophrenia when the clinician is skilled in the therapy and the patient has been carefully selected [10].

The PPCC Theory may be applied theoretically to compare schizophrenic, schizoaffective and non-psychotic patients who are addicted to substances. This theoretical extension compares the three categories of patient (Table 1). The three categories of patient are described visually according to the PPCC Theory in Figures 1, 2 and 3.

This theoretical proposition of the PPCC Theory suggests that Representations may be utilized as a variable that could open possibilities assisting recovery from addiction to both non-psychotic and psychotic patients if sufficient supportive and maintenance care is dedicated to the patients with a devoted, socially supportive, community spirit. Figures 1 and 2 show that Representations are the variable that is not affected overwhelmingly by the addiction, in the two milder groups of patients. The Problems variable at the top of all three diagrams consists of the problems given to the patient by their addiction. The Problems variable in Figures 2 and 3 also includes the problems given to the primarily mentally ill patients suffering from schizoaffective disorder and schizophrenia. Thus the problems caused by the illnesses schizophrenia and schizoaffective disorder include confusion, depression, social isolation, financial problems, accommodation difficulties and potential destitution. Potential

Table 1. Comparison of three kinds of patients who are addicts in relation to their mental health.

(i) (ii) (iii)

Psychiatric diagnosis:

Non-psychotic addict Schizoaffective addict Schizophrenic addict

Psychoanalytic understanding:

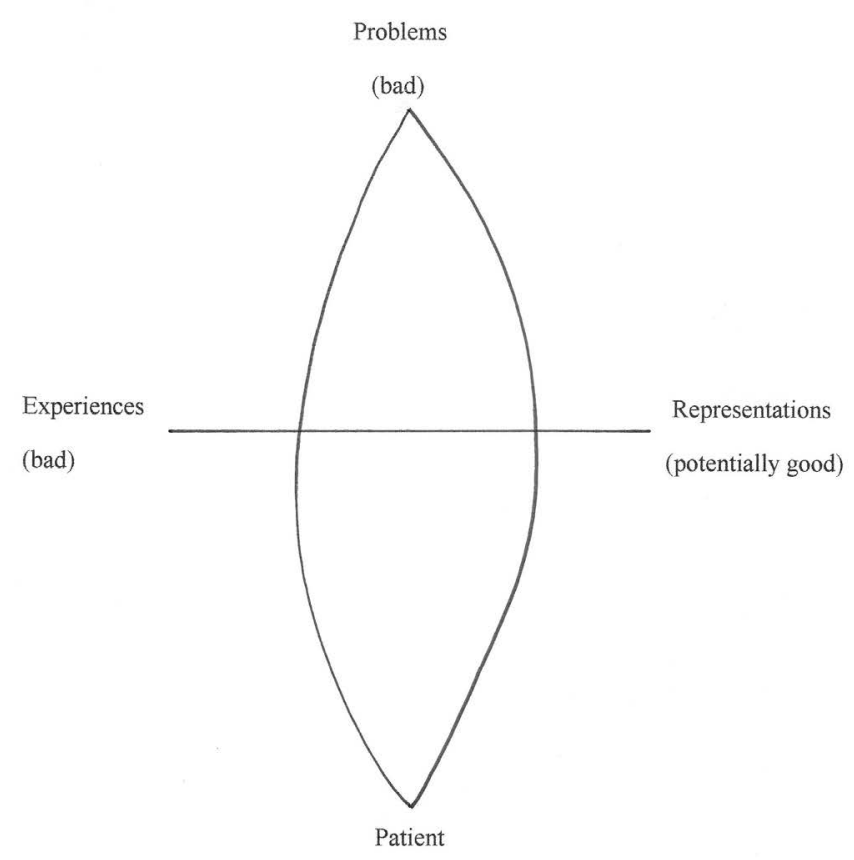

(integrated: potentially good)

Figure 1. Illustration according to the PPCC Theory of the Problems of addiction (but not of underlying psychosis) of an addicted non-essentially psychotic individual, in the depressive position.

Adapted with kind permission from BMJ case Reports Doi: 10.1136/bcr-2012-006683

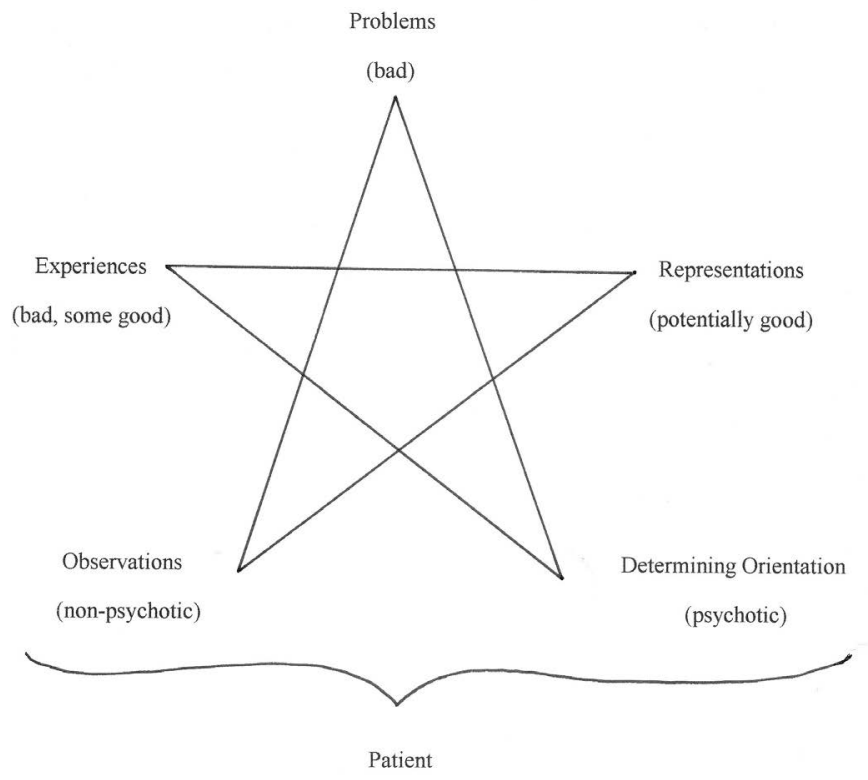

(split between psychotic and non-psychotic parts of mind: weakened)

Figure 2. Illustration according to the PPCC Theory of the Problems of addiction and underlying psychosis of an addicted schizoaffective individual, in the paranoid- schizoid position, untreated.

Adapted with kind permission from BMJ case Reports Doi: 10.1136/bcr-2012-006683.

problems relating to addiction include confusion, depression, lethargy, social isolation, financial problems, accommodation problems, destitution, hepatitis, AIDS and septicaemia. The Experiences variable contains the enjoyable aspects of the addiction ie. some good trips and euphoric states, but also all the bad experiences of every kind 

others

consequent upon the addiction. The non-psychotic Observations variable variously is integrated or not integrated with the psychotic Determining Orientation variable, thus enabling the patient to tackle their addiction or causing great difficulty in doing so, respectively; thus in the normal individual it is healthily integrated in Melanie Klein's depressive position, permitting the individual's mobilization into accessing all their self-sustaining resources in tackling their addiction. But in the schizoaffective individual the Observations variable is split from the psychotic Determining Orientation variable, in the paranoidschizoid position, weakening the patient and making it more difficult for them to tackle as an integrated individual their addiction; in this state, the patient is no match for the powerful addiction at Problems. And in the schizophrenic patient the Observations variable is conflated with the bad Experiences and completely blocked from the Dreams/ Representations variable (the Dreams component is not active in Figures 1, 2 or 3) and psychotic Determining Orientation variable; the patient is entirely incapable of tackling their addiction without help. Here, too, in the schizophrenic patient, the Representations variable is caught up in the blockage of the patient's mind and is scarcely accessible to treatment.

It can be seen that in the two cases of relatively milder ill-health, i.e., non-psychotic and schizoaffective patients, the only remaining, relatively inactive variable is the Dreams/ Representations variable. In the case of the schizophrenic patient who is addicted, the Dreams/ Representations variable is submerged into inaccessibility. But in the two milder cases, the Dreams/Representations variable is not otherwise engaged in the patient's mind. It could be utilized to help the patient tackle their addiction. Thus the PPCC's remaining unexpressed variable in its model of a schizophrenic or schizoaffective addict's mind, where most of the variables are compromised by mental illness or addiction, is Dreams/Representations. So, theoretically, if the Dreams/Representations variable could be made positively influential in the patient's life, this is predicted by the PPCC Theory to be a potentially very positive therapeutic tool. (For the schizophrenic

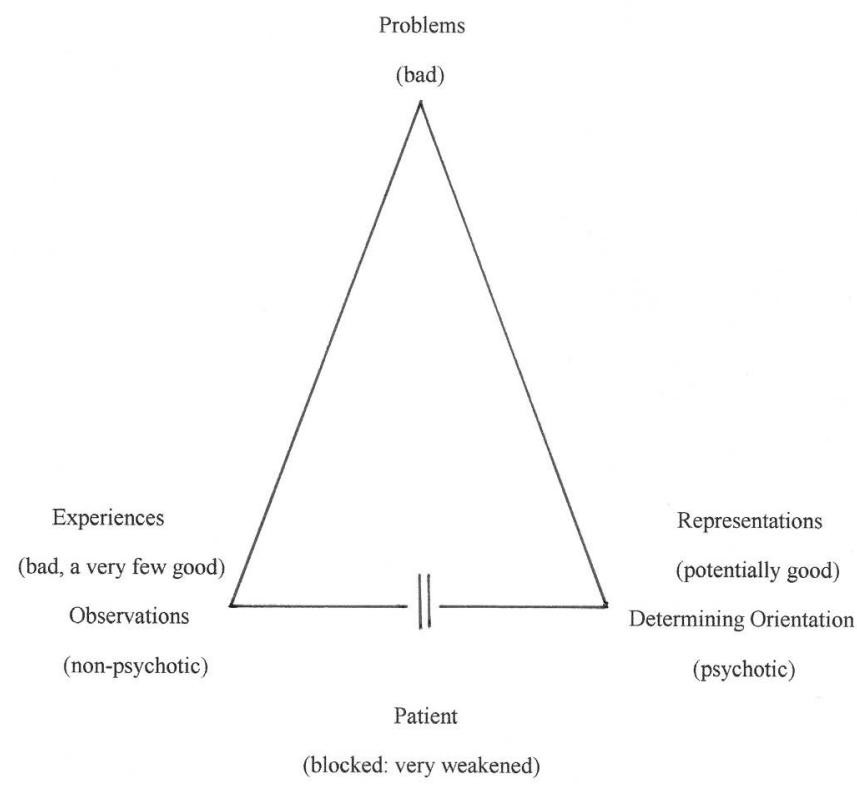

Figure 3. Illustration according to the PPCC Theory of the Problems of addiction and underlying psychosis of an addicted schizophrenic individual, in an entirely blocked position, untreated.

Adapted with kind permission from BMJ case Reports Doi: 10.1136/bcr-2012-006683 patient, firstly the addiction needs to be treated with the mild electric current or drugs and resolved, and then the schizophrenia treated; and then the Representational variable may become accessible as in the schizoaffective and normal patients, and allow the patient to adopt a Cherie, and recover).

The Representations variable could be utilized to represent symbolically Good images, representations, symbols, ideas, attitudes, examples and strengthening aspects of the patient's life, in their mind. In Figures 1, 2 and 3 it can be seen that the patient's Problems and Experiences are Bad and cause difficulties for the patient, but if the patient was enabled and encouraged to attend to Good, positive, wonderful, appealing, inspiring Representations, they might be able to commit to Good aspects of their life, rather than allowing the Bad aspects of their addiction to affect adversely their lives and preventing themselves from overcoming or dealing effectively with their psychotic mental illness. If the patient adopted a Distracting Investment or "Cherie", an extension of the PPCC Theory for Addictions, and personally committed their life to pursuit of this goal rather than their drug habit, a way might be found for them to develop a new identity for themselves away from their life of the drug habit.

The Cherie ("loved one" or "second bite at the cherry") would be a worldly concept that replaced all their negative, painful, distressing, frightening emotions, experiences and memories with regard to a positive, possible future for themselves. The patient would have to commit to it absolutely wholeheartedly themselves, but would be strongly supported in this endeavour by health, social and community support staff at a health centre while they transferred their allegiance from drugs to their Cherie. The patient would take in all aspects of their Cherie, and would engage fully and actively in pursuing their knowledge and understanding of it as a core part of their life. Examples of Cheries might be: engagement with colours and artwork, appraising others' work, painting and producing their own artwork, using different art materials, taking up photography, designing clothes, going to galleries and art exhibitions, learning about the history of art, exploring biographies of artists, recognising national trends in art styles; or responding to music in a similarly global way, trying to play music themselves, experimenting with different instruments, listening to others play, going to gigs, concerts and performances and visiting clubs to hear live bands, finding an interest in particular performers, learning about the history of music, its cultural origins and national trends and characteristics, reading biographies of performers and composers, writing music, songs and lyrics themselves; or the patient could devote themselves to animals, for example taking up riding and trekking with horses, or lavishing their care and attention on a pet dog or cat and getting to know the animal well, learning about animals' needs, health and welfare and common illnesses, becoming active in animal rescue, learning about different breeds of their chosen animal, taking their pet to cat or dog shows, forming good relations with other cat or dog owners, training dogs for the blind, and finding the satisfaction in animals that previously they found in drug "highs", but much more safely. There is plenty of scope for increasing the scope and breadth of a patient's own chosen Cherie, which would become the meaning of their life in substitution for their drug of addiction. The distracting Cherie would become what is craved and beloved, rather than the drug. There would be zero tolerance of the drug, and the Cherie would become in a basic, commonsense way a huge, rewarding distraction from the drug that would take up all the former addict's time and attention, while the biological aspects of rejection of the substance were being managed. 

others

\section{Results}

Table 2 summarizes the results of this theoretical exposition of the psychology of persons addicted to chemical substances who also have primary psychotic psychopathology, and their treatment. Although symptoms will clearly overlap across the various stages of treatment, it is considered very much preferable for the addiction to be resolved before the psychosis is definitively treated. If electric current treatment is ineffective then drugs may have to be used to resolve the addiction. But all decisions should be agreed with the patient, or they are unlikely to be effective.

\section{Discussion and conclusions}

The Distraction Investment or Cherie symbolizes Good for the patient, in contrast to the Bad drugs of addiction. The theoretical identification by the PPCC Theory of the Cherie as a Representation of everything good is already instanced in practice by Alcoholics Anonymous in its identification of aspects of God to its members. It is a very good example of studying the representations of a symbol, idea, concept, and ideal, ie. God, where love of the representations of the symbol enables the addict to substitute these new truths for the highly damaging truth that some drugs can sometimes provide nice feelings but at a terrible cost. The newly adopted, healing representations of God include emotional, spiritual, behavioural, law-conforming, social, community, musical, theological, literary, self-developmental and personally evolutionary benefits (depending on one's own personal perspective). These representations of God provide a potentially very strong counteraction to the problems of alcohol in the AA members' lives. It is theorized by the PPCC model that devotion to a Cherie such as those mentioned above may similarly enable an addict to substitute it for their drug habit. This might be particularly helpful for an addict without a pre-existing mental illness such as a psychosis, but it could be helpful also for a schizoaffective or schizophrenic patient if given enough support in overcoming as much as possible of their debilitating illness. Some cases of schizophrenia do gradually remit, and schizoaffective disorder is a treatable condition where episodes occur in early adult life but gradually cease. A Cherie could be a useful component of a patient's overall management.

Table 2. Summary of a theoretical treatment plan for an addict with an underlying psychosis.
1. Treat:
(i)
addiction: a) mild electric current to the head or drug therapy
b) distracting investment (Cherie).

(ii) schizoaffective disorder and schizophrenia: a) medication
b) individual therapy
c) group/social therapies
d) nurturing management.

2. Heal:

I (i) ) a feeling of intense wellbeing from environmental support

+ (ii) ) leading to a sense of joy: (a) love for Cherie

(b) absence of withdrawal symptoms

(c) absence of problems from drug habit.

Then:

II A new interest preoccupying whole life: Cherie.

Then:

III Diversify interests and flourish in life more broadly.
A conceptually and therapeutically useful aspect of Representations as a variable of the PPCC model is that a representation inter-relates internal awareness to the external world. A symbol of the outside world's reality may come to be part of an individual's internal life. And, equally, internal features of a person's self and mind may relate to features or phenomena about the external environment. Considering AA, a life in God's world may be said to relate internal virtues to good features about the surroundings of a person's life among the places and people they encounter. Regarding the Cheries, art and music create external beauty which may relate to internal appreciation and reference to a previous or existing sense of beauty. A pet may be truly beloved, linking the external world to wonderful personal expressions of contact, belonging, connection, meaning and joy. The scope of Representations is perhaps best illustrated by the deity, but the examples of Cheries mentioned could well fulfil a patient's search for meaning in life other than their drug of addiction.

Karl Schmidt has found [4] that a very weak electric current, with frequencies between $10-200 \mathrm{~Hz}$, applied to the back of the head (neuro-electric therapy, NET, devised by Dr Margaret Patterson) can eliminate chronic withdrawal symptoms in a patient relinquishing a substance of abuse without using other drugs. NET is developed from transcutaneous electro-nerve stimulation (TENS), and when used to treat pain is called brain electro-stimulation transcutaneously (BEST), or Trans Cranial Electro-Stimulation (TCES). None of the above involve electric shocks. Schmidt writes that "if we try to reduce one drug dependency by using another or the same drug (eg. methadone or heroin) which prevent the production and release of our own opioids [endorphins], our addiction most likely remains and it takes a long time to overcome. ......[But] with the use of an extremely low current applied to acupressure points on the back of the head, the production of natural opioids can be stimulated and the body encouraged to release its own opioids once more [which are suppressed by drugs of addiction eg. nicotine and alcohol]. We can then start on the road to non-addiction with a natural "feel good factor" produced by our own body." Schmidt argues that this mild electric current treatment permits healing of addictions without drugs or "cold turkey" withdrawal effects either.

The NET treatment takes about 10 days to complete, and has been found by some patients and staff to be very effective. Dr John Strang, a sceptic of the NET electric current therapy, wrote a paper on its application in 1984, which was not wholly unfavourable towards NET. But NET has not been studied formally in a randomised controlled trial to date, so formal results of its efficacy are not yet available (in 2016). Alternatively, according to preference or efficacy, drugs may be used to assist withdrawal from an addiction, for example acamprosate or disulfiram for alcohol withdrawal, nicotine replacement therapy or bupropion for nicotine dependence, and methadone or buprenorphine as opioid substitution therapy for opioid dependence.

Thus the combination of (1) mild electric current therapy or drugs to assist withdrawal, (2) devoted community support, (3) a beloved chosen Cherie or Distracting Investment, rewarding emotional strength and drive and representing values that are highly meaningful for the patient, and (4) psychological therapy, would appear to offer theoretically a good treatment regime to cure addictions in psychotic patients. Table 2 summarizes a theoretical treatment plan that combines treatment of the addiction and of the psychotic illness. It has been observed, as already mentioned, that psychosis is more likely to be caused by drug addiction than vice versa ${ }^{2}$, so treatment of the addiction should precede treatment of the core psychopathology. Treatment of schizoaffective 

others

disorder and schizophrenia require their own specific treatments, both individual and group therapy, which almost certainly should only be commenced once the addiction has been overcome. Examples of available therapies for schizophrenic and schizoaffective patients are group psychodynamic psychotherapy, avatar therapy, family therapy, social skills therapy, anticipatory skills therapy, cognitive behavioural therapy, community and social support, self-help groups, and physical health treatment by physicians, surgeons and dentists. Occasionally, schizoaffectively disordered patients may recover using psychoanalytic psychotherapy, as has been found with some schizophrenic patients. The above suggestions would theoretically seem, therefore, to offer a good opportunity for addicted persons with a psychotic illness and a range of needs to recover their mind.

\section{Funding}

\section{None}

\section{Competing interests}

\section{None}

\section{Acknowledgement}

Figures 1,2 and 3 are adapted with kind permission of BMJ Case Reports from "The process of recovery of a schizoaffectively disordered mind: a psychoanalytic theory of the functional psychoses, the psychodynamic pentapointed cognitive construct theory", G. Steggles (2012), doi:10.1136/bcr-2012-006683.

\section{References}

1. Andrews A, Knapp M, McCrone P, Parsonage M, Trachtenberg M (2012). Effective interventions in schizophrenia, the economic case: A report prepared for the Schizophrenia Commission. London: Rethink Mental Illness.

2. Meyer R (1986) Psychopathology and Addictive Disorders. New York, London: The Guilford Press.

3. Murray R, Quigley H, Quattrone D, Englund A, Forti MD, et al. (2016) Traditional marijuana, high-potency cannabis and synthetic cannabinoids: increasing risk for psychosis. World Psychiatry 15: 195-204. [Crossref]

4. Schmidt K (2008) Healing addictions without drugs (and no cold turkey either - a neurobiological approach), 5th Edition. UK: Sable Publishing House.

5. Steggles G (2012) The process of recovery of a schizoaffectively disordered mind: a psychoanalytic theory of the functional psychoses, the psychodynamic pentapointed cognitive construct theory. BMJ Case Reports.

6. Gelder M, Harrison P, Cowen P (2006) Shorter Oxford Textbook of Psychiatry. New York: Oxford University Press.

7. Robbins M (1993) Experiences of Schizophrenia. New York: The Guilford Press.

8. Klein M (1946) Notes on some schizoid mechanisms. In: Envy and Gratitude and other works 1946-1963. London: Hogarth Press, 1975:14. Int J Psychoanal 27: 99-110. [Crossref]

9. Klein M (1935) A contribution to the psychogenesis of manic-depressive states. In: Love, Guilt and Reparation and other works 1921-1945. London: Vintage, 1975:276. Int J Psycho-Anal 16: 145-174.

10. Steggles GR (2015) Stages in the psychological resolution of schizophrenia. Front Psychol 6: 86. [Crossref]

Copyright: (C2016 Steggles GRM. This is an open-access article distributed under the terms of the Creative Commons Attribution License, which permits unrestricted use, distribution, and reproduction in any medium, provided the original author and source are credited. 